\title{
EFFICIENT FATIGUE LIFE ASSESSMENT OF COMPOSITE MATERIALS BY USING A HYBRID SURROGATE MODELING
}

\author{
PRIMA P. AIRLANGGA \\ Department of Materials and Metallurgical Engineering, \\ Institut Teknologi Sepuluh Nopember, \\ Kampus ITS Keputih Sukolilo, 60111, Surabaya, East Java, Indonesia \\ primaairlangga@ymail.com \\ AZZAH D. PRAMATA \\ Department of Materials and Metallurgical Engineering, \\ Institut Teknologi Sepuluh Nopember, \\ Kampus ITS Keputih Sukolilo, 60111, Surabaya, East Java, Indonesia \\ azzah@its.ac.id \\ MAS IRFAN P. HIDAYAT* \\ Department of Materials and Metallurgical Engineering, \\ Institut Teknologi Sepuluh Nopember, \\ Kampus ITS Keputih Sukolilo, 60111, Surabaya, East Java, Indonesia \\ irfan@mat-eng.its.ac.id \\ * Corresponding author
}

Received 1 December 2021; Published 31 December 2021

\begin{abstract}
In this study, hybrid surrogate and nonlinear autoregressive with exogenous inputs (NARX) model is developed and presented as data-driven based predictive model for efficient fatigue life assessment of composite materials. Surrogate modeling based upon wavelet neural networks (WNN) is employed to efficiently unveil mathematical pattern in S-N data, but costly to get from experiments. Moreover, the NARX architecture is chosen in order to enable multi-step ahead prediction in fatigue life assessment of multivariable amplitude loadings. By observing fatigue data as dynamic data of stress ratio series, it is shown that the hybrid model produces reasonably accurate fatigue life prediction by using fatigue data from two stress ratio values only. The use of two stress ratio values also allows usage of more limited fatigue data in the lifetime prediction. The WNN-NARX surrogate model is tested with well-known fatigue data in literature. Several composite materials examined in this study show the efficacy and robustness of the proposed model.
\end{abstract}

Keywords: surrogate model; fatigue life prediction; limited fatigue data; multivariable amplitude loading; composite materials.

\section{Introduction}

Together with increasing use of composite materials in various important and critical engineering applications, there has been also increasing needs for more reliable evaluation and assessment of their lifetime in services and operations. To that end, several fatigue life models for composite materials have been introduced and proposed in the last decades (Reifsnider, 1991; Harris, 2003). Fatigue life models concerning with composite materials may be categorized into several types: laminate modeling based on damage evolution (Lian and Yao, 2010), multiaxial fatigue damage model (Kennedy et al., 2013), fatigue damage accumulation based on stiffness degradation (Shiri et al., 2015), phenomenological approach accounting for mean stress effect (Flore and Wegener, 2016), residual strength degradation modeling (Stojković et al., 2017) as well as multiaxial fatigue life prediction based on fiber-scale unit model (Weng et al., 2017). Due to the fact that many fatigue life models are relying on fatigue data in the form of stress-life/cycle or S-N data as a key ingredient of the model, efficient evaluation of the fatigue data therefore still becomes main concern and interest. On the other hand, driven by needs to deal with more dynamic and challenging operating conditions, interests in fatigue life assessment for a 
component or structure under spectrum loadings (Vassilopoulos et al., 2008; Yaich et al., 2017) are greatly increasing in recent years. In this regard, constant life diagrams (CLD) has been shown to be a useful tool for such lifetime assessment (Sendeckyj, 2001; Freire Junior et al., 2005). As the CLD is essentially another representation of S-N data, it is obvious that efficient inquiry of S-N data is noteworthy for efficient fatigue life assessment of composite materials, in particular for multivariable amplitude or spectrum loadings.

In recent years, attempts have been made so that $\mathrm{S}-\mathrm{N}$ data can be obtained more efficiently within reasonable time and cost (Durodola et al., 2017; Kalombo et al., 2020). For composite materials, the effort is more challenging. It is costly to collect all fatigue data experimentally by taking all variables into account including fiber orientation, laminate/lay-ups design, loading conditions (Kang et al, 2006; Chen and Shang, 2011), mean stress effect (Al-Assadi et al., 2011; Durodola et al., 2018) as well as types of fiber/matrix, including manufacturing process (Yang et al., 2020; Shanmugam et al., 2021). Recently, many researchers also route to machine learning approaches to address the problems efficiently. The approaches range from neural networks (NN), polynomial classifiers and recurrent NN, modular networks, NN with Bayesian regularization, cumulative distribution function model and genetic programming (Bucar et al., 2006; Bezazi et al., 2007; Vassilopoulos et al., 2008; Pujol and Pinto, 2011). See more recent reviews on fatigue modelling using soft computing methods (Kalayci et al., 2020; Chen and Liu, 2021). Advantages from the approaches seem to be promising. For instance, reduced number of fatigue tests could be reasonably achieved without sacrificing or impairing accuracy in the assessment process. It also appears that the route has been opening possibilities for further exploration of other advantages, especially for building efficient data-driven based predictive models (Pais et al., 2012; Zhu et al., 2019; Song et al., 2019) which are able to capture important information behind the underlying physical mechanisms from the observed data, while having statistical justification at the same time. This research work is motivated by the previous works and particularly in order to achieve more robust and efficient fatigue life assessment of composite materials.

In the present work, hybrid surrogate and nonlinear autoregressive with exogenous inputs (NARX) model is developed and presented as data-driven based predictive model for efficient fatigue life assessment of composite materials. Surrogate modeling based upon wavelet neural networks (WNN) is employed to efficiently unveil mathematical pattern in S-N data, but costly to get from experiments. Moreover, the NARX architecture is chosen in order to enable multi-step ahead prediction in fatigue life assessment of multivariable amplitude loadings. The fatigue life assessment presented in this study highlights the following key aspects: (i) stable datadriven based predictive models with regression inputs which allow the use of limited fatigue data i.e. those belong to two stress ratio values. As a result, the experimentation cost for collecting fatigue data can be reduced significantly, (ii) the predictive models are robust i.e. less preference is needed to select the available fatigue data to be used as basis/training data. This allows quick fatigue life prediction based upon available limited data in hands, (iii) the assessment is able to cover multivariable amplitude fatigue loadings of tension-tension (T-T), tension-compression (T-C) and compression-compression (C-C). It therefore constitutes to wide spectrum of fatigue loadings and leading to an efficient way to perform fatigue life assessment of composite materials under multivariable amplitude loadings (thus suitable for building constant life diagrams (CLD) efficiently as well), and (iv) the assessment provides lower and upper bounds of prediction, giving prediction interval integrated with the assessment and scattered nature of S-N data (Schijve, 2009; Klemenc and Fajdiga, 2012).

In this study, the WNN (Zhang and Benveniste, 1992) has been employed in combination with NARX structure to yield an integrated surrogate model and enabling more enhanced generalization ability in the light of limited training data (Hidayat and Yusoff, 2009; Hidayat, 2015). To the present author's best knowledge, the advantage of using NARX as an integrated approach in fatigue life assessment of materials is still less explored and limited in literature. It is worth to note that by observing the fatigue data as dynamic data, the present study leads to multi-step ahead prediction, which is shown to be an effective and efficient approach for fatigue life assessment of composite materials. The WNN-NARX surrogate model is tested with well-known fatigue data in literature. The simulation results show that the proposed surrogate model is robust with good accuracies for efficient fatigue life assessment of composite materials under multivariable amplitude loadings.

This paper is organized as follows: surrogate modeling and the WNN as the surrogate model are presented in section 2. In addition, the observation as basis for treating fatigue data as dynamic data leading to the use of NARX structure in combination with the WNN is also given in this section. It is followed by section 3 of materials and methods, including the steps of preprocessing, initialization of networks as well as processing and prediction using the WNN. Results are presented in section 4, followed by conclusions in section 5. 


\section{Surrogate Modelling}

Surrogate modeling is a technique of approximating a function which is expensive to evaluate with another one which is cheaper or more convenient to evaluate (Kleijnen, 2009; Martin and Simpson, 2005; Han et al., 2010). A surrogate model is also known as a meta-model i.e. the model of an underlying model of function. For optimization problems, it can be considered as an approximation model for cost function/state function and built from sample data obtained by randomly probing or sampling the design space of interest. In contrast to deterministic models, the surrogate models take bias produced from the approximation of underlying model into account. The meta-model is thus containing of stochastic part of the approximation, in addition to its deterministic one, as follows (Prebeg et al., 2014):

$$
y(\mathbf{x})=\hat{y}(\mathbf{x})+\varepsilon(\mathbf{x})
$$

where: $y(\mathbf{x})$ is a function or response of interest observed at a set of $p$ data points/observations called experimental design, $\hat{y}(\mathbf{x})$ is a surrogate model of response $y(\mathbf{x}), \varepsilon(\mathbf{x})$ is the model error or bias. The error represents both the error of approximation due to lack of fit of the surrogate model and the random error due to intrinsic noises, and $\mathbf{x}$ is a vector of input variables.

The process for constructing the model based upon available data in hands is called as fitting or training process and the dataset is called as sampling points or training points/patterns (Hwang and Martins, 2018; Tripathy and Bilionis, 2018). Through the fitting/training process, the objective of a surrogate model is to compute weight parameters $\mathbf{W}$ so that:

$$
\hat{y}_{i}=f\left(\mathbf{x}_{i}, \mathbf{w}\right), \quad 1 \leq i \leq p
$$

by minimizing a cost function of residuals/mismatch between the prediction $\hat{y}_{i}$ and the correct output $y_{i}$ or optimizing the likelihood function (Scheuerer et al., 2013). The commonly used cost or objective function to be minimized is:

$$
E=\frac{1}{2} \sum_{i=1}^{n}\left[y_{i}-\hat{y}_{i}\right]^{2}
$$

with $n$ the number of training data or samples. In this work, WNN is employed as surrogate models through which the modelling of fatigue life assessment of composite materials is carried out. efficiently The WNN is explained in what follows.

\subsection{Wavelet Neural Networks}

Neural networks (NN) have been established as a general approximation tool for fitting nonlinear models from input/output data. WNN is a class of feedforward NN having multidimensional wavelets $\psi$ constructed from a mother wavelet.

The hidden neuron in WNN is called as wavelon. In contrast to NN, rather than fitting weights only, all parameters of weights, dilations and translations will be jointly fitted from the training data by the WNN. The approximation by the WNN to realize (2) is expressed as:

$$
\hat{f}(\mathbf{x})=\sum_{i=1}^{N} \mathbf{w}_{i} \psi\left[\mathbf{D}_{i} \mathbf{R}_{i}\left(\mathbf{x}-t_{i}\right)\right]+\bar{g}
$$

where: $t$ translation parameter, $\mathbf{D}$ the dilation matrices built from the dilation vectors, $\mathbf{R}$ the rotation matrices, $\bar{g}$ the additional parameter (bias) at the output layer and $N$ the number of hidden neurons (wavelons). As more parameters in the WNN need to be adjusted compared to those in NN, a stochastic gradient algorithm with projections is preferred than usual gradient based or Newton algorithms for computational saving when proceeding with the minimization of objective function (3). The choice of training algorithm keeps the computation efficient while preserving the accuracy of prediction. In addition, as the mother wavelet, the following wavelet function is chosen:

$$
\psi(\mathbf{x})=-\mathbf{x} e^{-\frac{1}{2} \mathbf{x}^{2}}
$$


Different with other types of NN like multilayer perceptron (MLP) NN which uses discriminatory functions (sigmoid functions) and radial basis functions (RBF) NN which uses distance/radial functions in their approximations, the WNN uses multiresolution analysis based on wavelet transform (Mallat, 1989; Daubechies, 1992). For clarity of presentation, Fig. 1 depicts schematic of a WNN.

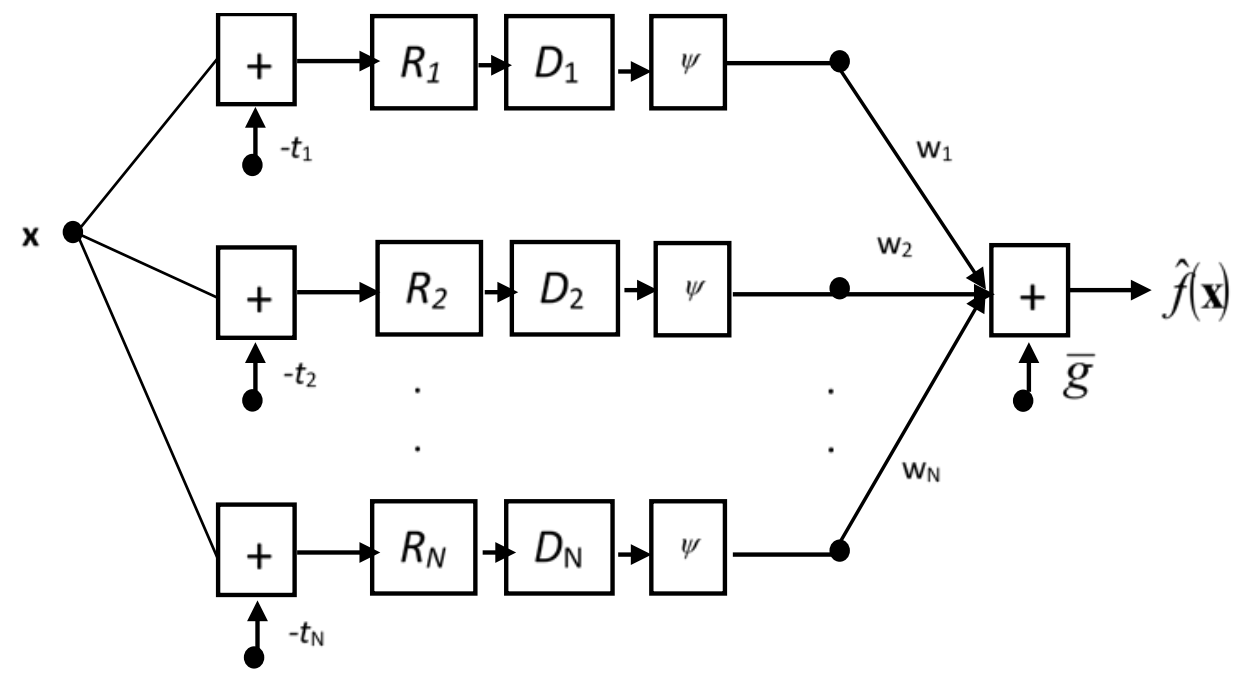

Fig. 1. Schematic of WNN.

\subsection{Nonlinear Autoregressive with Exogenous Inputs (NARX)}

Fatigue data may be viewed as dynamic data, rather than as static data, although there is no time variable explicitly. This can be observed when one transforms the S-N data into another representation i.e. CLD, in which one can move from tension-tension (T-T), tension-compression (T-C) and compression-compression (C-C) regions in the diagram. In other words, there is transition between the regions which is represented by different stress ratio $R$ values. Lines representing the stress ratios in the CLD as well as the isolines of fatigue life can then be plotted and made denser based on the requirement in hands. In such representation, fatigue data can be viewed as dynamic data with respect to the transition of stress ratio values. For completeness, the schematic of CLD is shown in Fig. 2.

Based on the observation, the structure of nonlinear autoregressive with exogenous inputs (NARX) is chosen in this study and further applied to the fatigue data which are now seen as stress ratio series. The NARX structure can be expressed as (Chen et al., 1990; Narendra and Parthasarathy, 1990):

$$
y(n+1)=f[\mathbf{y}(n) ; \mathbf{u}(n)]
$$

$$
y(n+1)=f\left[y(n), \ldots, y\left(n-d_{y}+1\right) ; u(n), u(n-1), \ldots, u\left(n-d_{u}+1\right)\right]
$$

where: $u(n)$ and $y(n)$ state respectively the input and output of the NARX model at discrete time $n$ and $u(n), y(n)$ $\epsilon \mathfrak{R}$. Also, $d_{y}$ and $d_{u}$ are the output-memory and input-memory orders. $d_{y}$ represents the number of lagged output values, which is often referred to as the order of the model, $d_{u}$ represents the number of lagged input values $\left(d_{u}\right.$, $d_{y} \geq 1$ and $\left.d_{u} \leq d_{y}\right)$. The vectors $\mathbf{y}(n)$ and $\mathbf{u}(n)$ form the output and input regressors, respectively.

The NARX model is commonly trained using two basic modes i.e. parallel (P) mode and series-parallel (SP) mode. In the $\mathrm{P}$ mode, the regressors employs the estimated outputs which are fed back to the regressor, as follows:

$$
\hat{y}(n+1)=\hat{f}\left[\hat{y}(n), \ldots, \hat{y}\left(n-d_{y}+1\right) ; u(n), u(n-1), \ldots, u\left(n-d_{u}+1\right)\right]
$$

On the other hand, the regressors use the actual output values in the SP mode, given as: 


$$
\hat{y}(n+1)=\hat{f}\left[y(n), \ldots, y\left(n-d_{y}+1\right) ; u(n), u(n-1), \ldots, u\left(n-d_{u}+1\right)\right]
$$

Based on the description, the P and SP architectures are hence suitable for online and offline modes of learning, respectively. The NARX structures with input and output tapped delay lines (TDL) in P and SP modes (Neural Network Toolbox ${ }^{\mathrm{TM}}$ User’s Guide, 2017) are illustrated in Fig. 3.

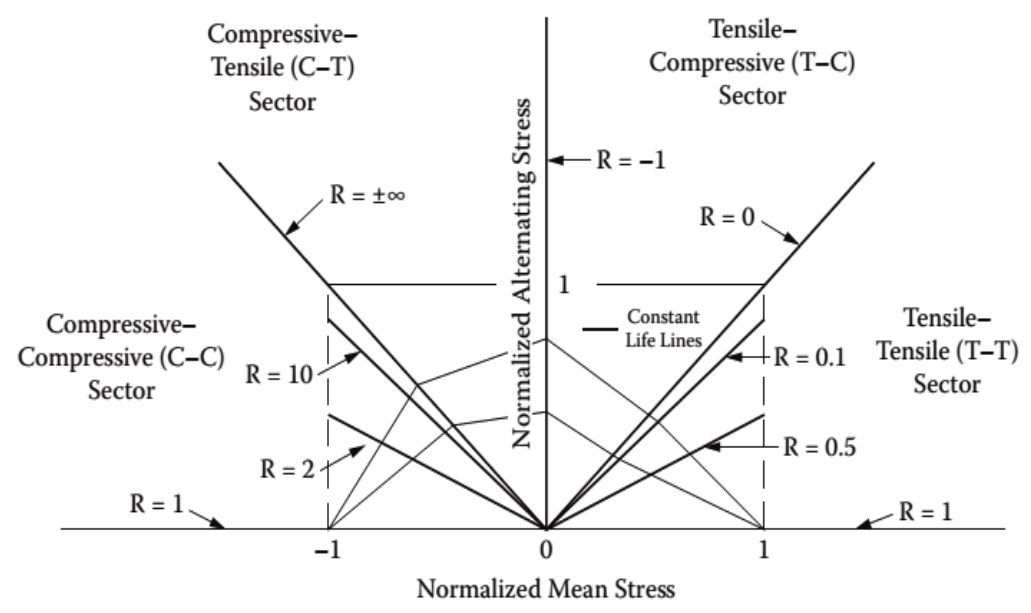

Fig. 2. Schematic of CLD for fatigue life assessment and analysis tool. The fatigue data may be now seen as stress ratio series.

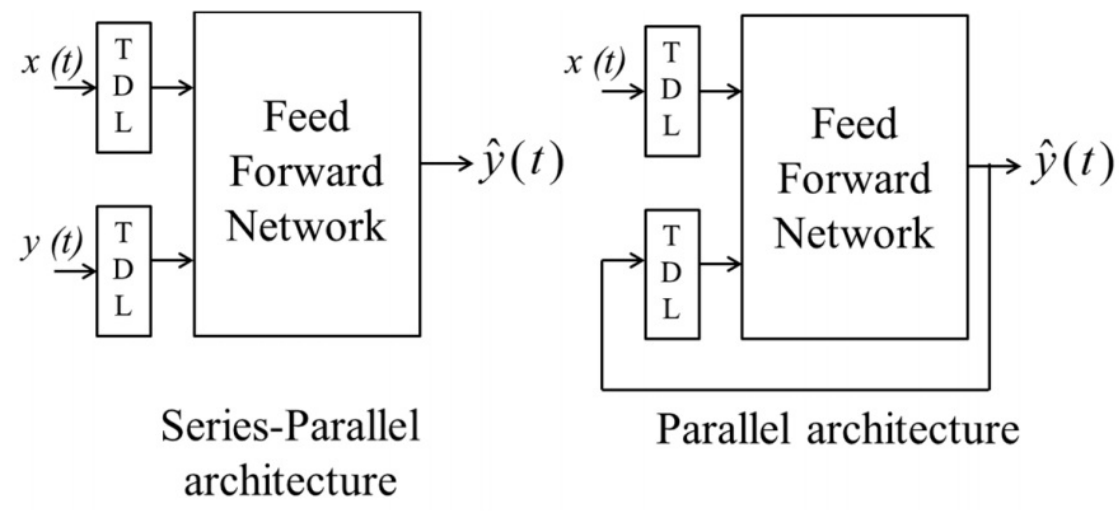

Fig. 3. P and SP modes of NARX model [47].

It is also noted here that in contrast to the previous studies (Hidayat and Yusoff, 2009; Hidayat, 2015) in which the standard feedforward NN or the MLP-NARX models were employed with the gradient based training algorithms: (i) there is no need to use explicit form of regularization for the objective function with the present hybrid WNN-NARX model for handling the noise in data, (ii) it is not necessary to determine the number of hidden nodes/neurons in advance, as the number of wavelons is started, adjusted and fixed during the networks running based upon the training data/samples given, and (iii) the present hybrid model allows multi-step ahead 
prediction of fatigue data at other different stress ratio values based upon limited fatigue data coming from two stress ratios as training data. The use of limited fatigue data as the training data is justified by looking at the whole fatigue data of different stress ratio values as the stress ratio series, mentioned previously.

\section{Materials and Methods}

\subsection{Materials}

Fatigue data from three different composite materials are assessed in the present study. The composite materials are E-glass/polyester $[90 / 0 / \pm 45 / 0]_{s}$, E-glass fabrics/epoxy $\left[ \pm 45 / 0_{4} / \pm 45 /\right]$ and E-glass/polyester $\left[0 /( \pm 45)_{2} / 0\right]$, commonly used in wind turbine blade applications. They are denoted as materials 1,2 and 3, respectively, where their fatigue data are available online (Mandell and Samborsky, 2010; Vassilopoulos and Philippidis, 2002). From the available fatigue data, a set of fatigue data from two stress ratios is used as the training samples, while the remaining fatigue data of other stress ratios are predicted with the present hybrid WNN-NARX model. Furthermore, in order to be consistent all the remaining stress ratios are arranged in sequence from that is lying in $\mathrm{T}$-T region to that is lying in CC-region of the CLD.

In literature, material 1 is called as DD16 [48]. Its database is consisting of fatigue data of various $R$ values $(R=0.1,0.5,0.7,0.8,0.9,-0.5,-1,-2$ and 10$)$ with on-axis orientation only. Having 5 stress levels for each stress ratio, the material has 45 fatigue data. On the other hand, material 2 has on-axis $\left(0^{\circ}\right)$ and off-axis $\left(90^{\circ}\right)$ orientations, from cutting by diamond saw wheel. Material 2 is also known as material QQ1 (Mandell and Samborsky, 2010). For material 3, the axis orientations are $0^{\circ}$ and $45^{\circ}$ (Vassilopoulos and Philippidis, 2002). Materials 2 and 3 are denoted as on-axis and off-axis for different loading orientations. By noting that number of stress levels for each stress ratio to be 8 and 5 for materials 2 and 3, respectively, the materials have 96 and 40 fatigue data in total, respectively.

Apart from the fact that materials 1, 2 and 3 have different on-and off-axis orientations, the simulation procedure for all materials is the same i.e. by separating fatigue data from two stress ratio values as the training data set. Moreover, the number of fatigue data used as the training data/samples for material 1 is $22.22 \%$, material 2 on-axis and material 2 off-axis are 33.33\%, respectively, and material 3 on-axis and material 3 offaxis are also 50\%, respectively, from their respective total fatigue data.

All training and testing sets for the materials are given in Tables 1-3 below. From Tables 1-3, it is obvious that the stress ratios of $R=0.1$ and 10 are in strategic positions as they represent opposite sectors in the CLD. Nonetheless, this option of training set is not mandatory. Other set of stress ratios can be chosen as well and not too restrictive, as will be shown in the next section.

Table 1. Material 1 along with the training and testing sets employed for its fatigue life assessment

\begin{tabular}{|c|c|c|c|}
\hline Material & $\begin{array}{c}\text { Angle } \\
\text { orientation }\end{array}$ & $\begin{array}{c}\text { Fatigue data as } \\
\text { training set from } R \\
\text { values }\end{array}$ & $\begin{array}{c}\text { Fatigue data as testing set from } \\
R \text { values }\end{array}$ \\
\hline $\begin{array}{c}\text { E-glass/polyester } \\
{[90 / 0 / \pm 45 / 0]_{\mathrm{s}}[48]}\end{array}$ & On-axis & $R=0.1$ and 10 & $R=0.9,0.8,0.7,0.5,-0.5,-1$ and -2 \\
\hline
\end{tabular}

Table 2a. Material 2 (on-axis) along with the training and testing sets employed for its fatigue life assessment

\begin{tabular}{|c|c|c|c|}
\hline Material & $\begin{array}{c}\text { Angle } \\
\text { orientation }\end{array}$ & $\begin{array}{c}\text { Fatigue data as training set from } \\
R \text { values }\end{array}$ & $\begin{array}{c}\text { Fatigue data as testing set from } \\
R \text { values }\end{array}$ \\
\hline $\begin{array}{c}\text { E-glass fabrics/epoxy } \\
{\left[ \pm 45 / 0_{4} / \pm 45 /\right]} \\
{[48]}\end{array}$ & On-axis & $R=0.1$ and 10 & $R=0.5,-0.5,-1$ and -2 \\
\hline
\end{tabular}


Table 2b. Material 2 (off-axis) along with the training and testing sets employed for its fatigue life assessment

\begin{tabular}{|c|c|c|c|}
\hline Material & $\begin{array}{c}\text { Angle } \\
\text { orientation }\end{array}$ & $\begin{array}{c}\text { Fatigue data as training set from } \\
R \text { values }\end{array}$ & $\begin{array}{c}\text { Fatigue data as testing set from } \\
R \text { values }\end{array}$ \\
\hline $\begin{array}{c}\text { E-glass fabrics/epoxy } \\
{\left[ \pm 45 / 0_{4} / \pm 45 /\right]} \\
{[48]}\end{array}$ & $\begin{array}{c}\text { Off-axis } \\
\left(90^{\circ}\right)\end{array}$ & $R=0.1$ and 10 & $R=0.5,-0.5,-1$ and -2 \\
\hline
\end{tabular}

Table 3a. Material 3 (on-axis) along with the training and testing sets employed for its fatigue life assessment

\begin{tabular}{|c|c|c|c|}
\hline Material & $\begin{array}{c}\text { Angle } \\
\text { orientation }\end{array}$ & $\begin{array}{c}\text { Fatigue data as training set from } \\
R \text { values }\end{array}$ & $\begin{array}{c}\text { Fatigue data as testing set from } \\
R \text { values }\end{array}$ \\
\hline $\begin{array}{c}\text { E-glass/polyester } \\
{\left[0 /( \pm 45)_{2} / 0\right]} \\
{[49]}\end{array}$ & On-axis & $R=0.1$ and 10 & $R=0.5$ and -1 \\
\hline
\end{tabular}

Table 3b. Material 3 (off-axis) along with the training and testing sets employed for its fatigue life assessment

\begin{tabular}{|c|c|c|c|}
\hline Material & $\begin{array}{c}\text { Angle } \\
\text { orientation }\end{array}$ & $\begin{array}{c}\text { Fatigue data as training set from } \\
R \text { values }\end{array}$ & $\begin{array}{c}\text { Fatigue data as testing set from } \\
R \text { values }\end{array}$ \\
\hline $\begin{array}{c}\text { E-glass/polyester } \\
{\left[0 /( \pm 45)_{2} / 0\right]} \\
{[49]}\end{array}$ & $\begin{array}{c}\text { Off-axis } \\
\left(45^{\circ}\right)\end{array}$ & $R=0.1$ and 10 & $R=0.5$ and -1 \\
\hline
\end{tabular}

\subsection{Preprocessing}

Data pre-processing is an important step for successful regression and prediction using NN in order to get better scalability of data. In this stage, all fatigue data are normalized so that the data fall in the range of -1 to 1 using the following normalization formula:

$$
x_{\mathrm{n}}=\frac{2 \mathbf{x}-x_{\max }-x_{\min }}{x_{\max }-x_{\min }}
$$

where: $\mathbf{x}_{n}$ is the normalized value of the input/output variables, $\mathbf{x}$ is the original data and $\mathbf{x}_{\min }, \mathbf{x}_{\max }$ are the minimum and maximum values of the variables, respectively.

Stress ratio $R$, maximum stress $\left(S_{\max }\right)$ and minimum stress $\left(S_{\min }\right)$ are used as the input variables for fatigue life prediction of the materials. The inputs are chosen as they are essential to describe fatigue and other stresses such as mean stress $\left(S_{\mathrm{m}}\right)$ and amplitude stress $\left(S_{\mathrm{a}}\right)$. The fatigue cycles in logarithmic $(\log N)$ corresponding to the input sets are used as the prediction target. The used value of fatigue cycle is the mean value of fatigue cycles for a stress level.

\subsection{Initialization of networks}

Network parameters of weights (including bias), dilation, rotation and translation need to be initialized for the WNN. For weights, they are initially set to be zero, while the estimated mean of the function to be approximated is used as the initial value for bias $\bar{g}$. For the dilation, rotation and translation parameters, the advantage of using WNN is that initialization of the parameters is proceeded based upon the given samples of noisy input/output observations $\{\mathbf{x}, f(\mathbf{x})\}$. It means that the parameters initialization and adjustment are driven by 
the inputted samples to the networks, hence requiring less intervention from user by fixing parameters much in advance like other types of feed-forward NN. In addition, the WNN can naturally handle noisy data without any form of regularization parameters like other feed-forward NN. The robustness of wavelet transform/compression for noise removal has been known in signal processing (Mallat, 1989; Daubechies, 1992).

Furthermore, another advantage of using the WNN is that the number of wavelons needed for the prediction task is adjusted or reduced automatically based upon the training samples fed to the network. One just needs to supply initial number of wavelons at the beginning. In this study, initial number of wavelons of 10 is used. Moreover, performance/error goal for the objective function (3) is by default set to be zero. That is the error is forced to be as small as possible within a given number of iterations. Lastly, maximum number of epochs or iterations needs to be set by the user. It is set to be 10 epochs as termination for stopping the iterations.

\subsection{Processing and prediction}

Given the training samples, the network will try to keep its structure/architecture as simple as possible with respect to the number of neurons/wavelons in order to do the prediction task. By keeping the number of wavelons in minimum, the iteration process for adjusting the network parameters will be kept simpler and easier as well. The way also keeps the robustness of prediction while saving the computational cost. There are three approaches which can be used by the WNN to maintain the model complexity i.e. residual based regressor selection, stepwise regressor selection by orthogonalization and backward elimination of regressor. In general, variables are added in sequence in the forward selection until no more improvement in the prediction and a number of regressors is obtained. On the other hand, all variables are chosen initially in the backward selection and then variables with less significance to the prediction are removed from the model. While, the stepwise selection is in between the forward and backward selection to produce an efficient acceleration of variables selection. Using the algorithm, variables can be brought into or removed from the model without affecting the other variable estimates. It is achieved by making the regressors orthogonal to eliminate their interactions. In this study, the second approach is chosen.

After the model selection process, the WNN continues to minimize the objective function (3). Suppose that all the parameters $w_{i}, \bar{g}, t_{i}, D_{i}$ and $R_{i}$ are collected into the parameter vector $\boldsymbol{\theta}$, then the parameter vector is modified to minimize the objective function following the stochastic gradient with projections algorithm. In the algorithm, the parameter vector is updated in the opposite/negative direction of the gradient of following error functional:

It proceeds as:

$$
c\left(\boldsymbol{\theta}, \mathbf{x}_{k}, y_{k}\right)=\frac{1}{2}\left[g_{\theta}\left(\mathbf{x}_{k}\right)-y_{k}\right]^{2}
$$

$$
\overline{\boldsymbol{\theta}}_{k}=\boldsymbol{\theta}_{\mathrm{k}-1}-\gamma_{k} \operatorname{grad} c\left(\boldsymbol{\theta}_{k}, \mathbf{x}_{k}, y_{k}\right)
$$

with $\gamma$ is the gradient learning parameter, commonly a small number $0<\gamma<1$ and constraints $C \in C$ are set on the adjusted parameters (dilation, rotation and translation parameters). The intermediate parameter vector $\overline{\boldsymbol{\theta}}_{k}$ is then projected to the parameter space $C$, which is convex, through the projection operator $P$ as follows:

$$
\boldsymbol{\theta}_{\mathrm{k}}=P\left(\overline{\boldsymbol{\theta}}_{k}\right)
$$

In contrast to common gradient descent algorithm, the stochastic gradient with projections algorithm improves the iteration in order to avoid to be trapped in local minimum. Once the prediction outputs are produced through the WNN, one needs to do un-normalization to the network outputs. The accuracy of prediction is then assessed with respect to the real fatigue lives $(\log N)$ from the experimental data.

\section{Results and Discussion}

Fatigue life predictions of the aforementioned composite materials with the proposed hybrid WNN-NARX model are carried out in MATLAB environment. The results are presented in the form of S-N curve for more clarity of presentation. The closeness between the fatigue lives as predicted by the present model and those from experiments is given in term of the coefficient of determination $\mathrm{R}^{2}$. 


\subsection{Fatigue life prediction of material 1}

Fatigue life prediction results on the testing set for material 1 are presented accordingly in Figs. 4-7. The S- $N$ curves are plotted by using the amplitude stress values to show the variability of loading amplitudes.

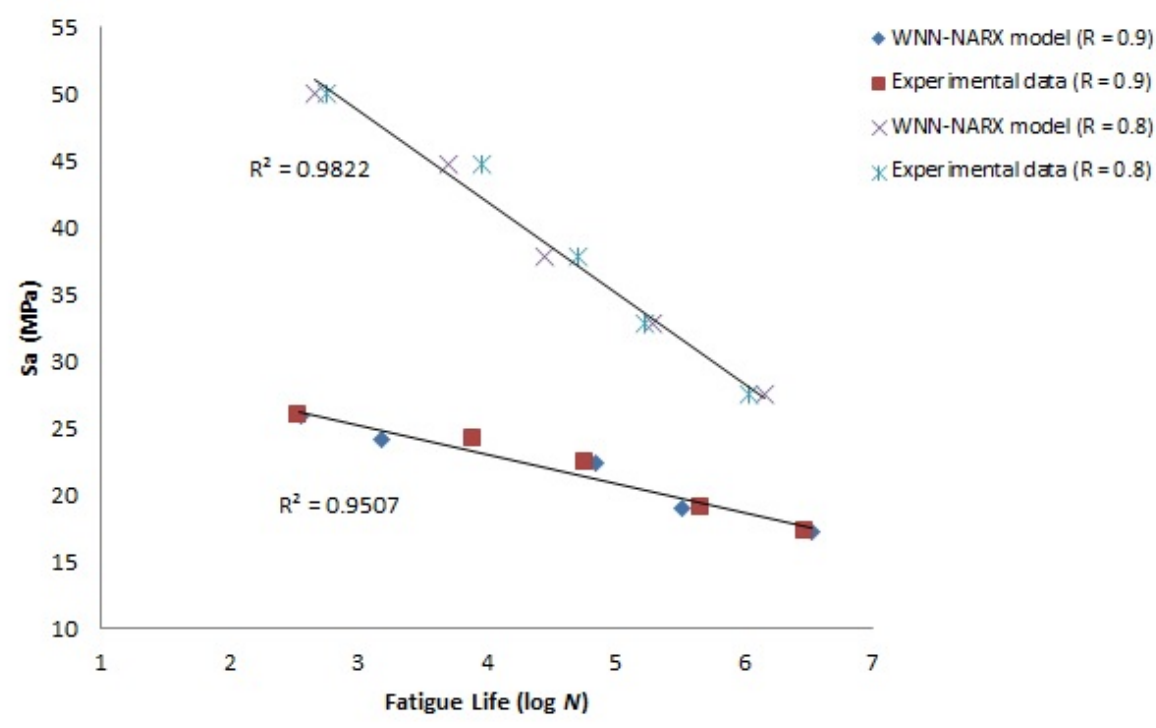

Fig. 4. Fatigue life prediction on the testing set of $R=0.9$ and 0.8 for material 1 .

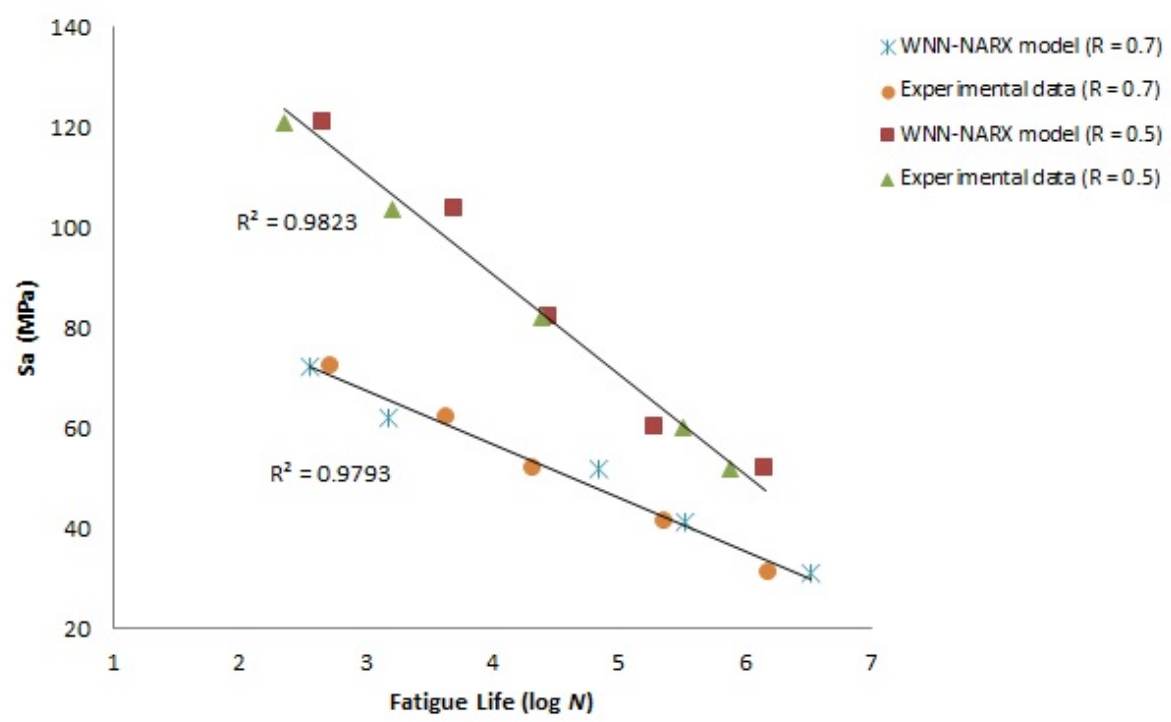

Fig. 5. Fatigue life prediction on the testing set of $R=0.7$ and 0.5 for material 1 . 


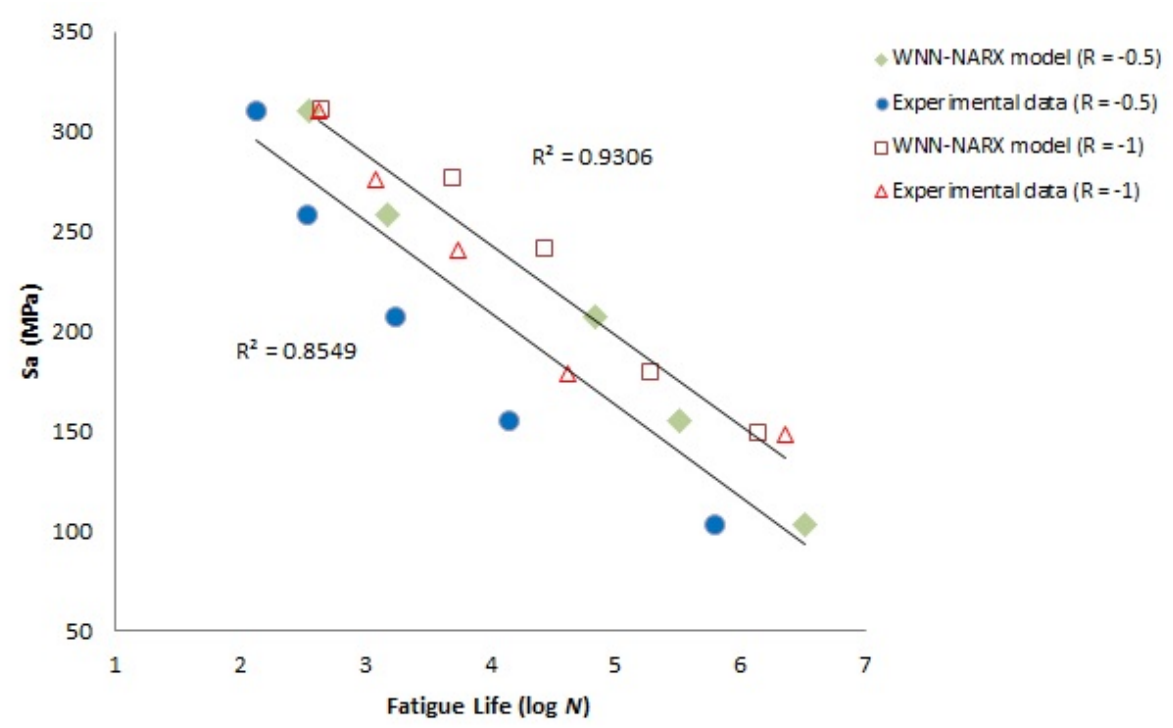

Fig. 6. Fatigue life prediction on the testing set of $R=-0.5$ and -1 for material 1 .

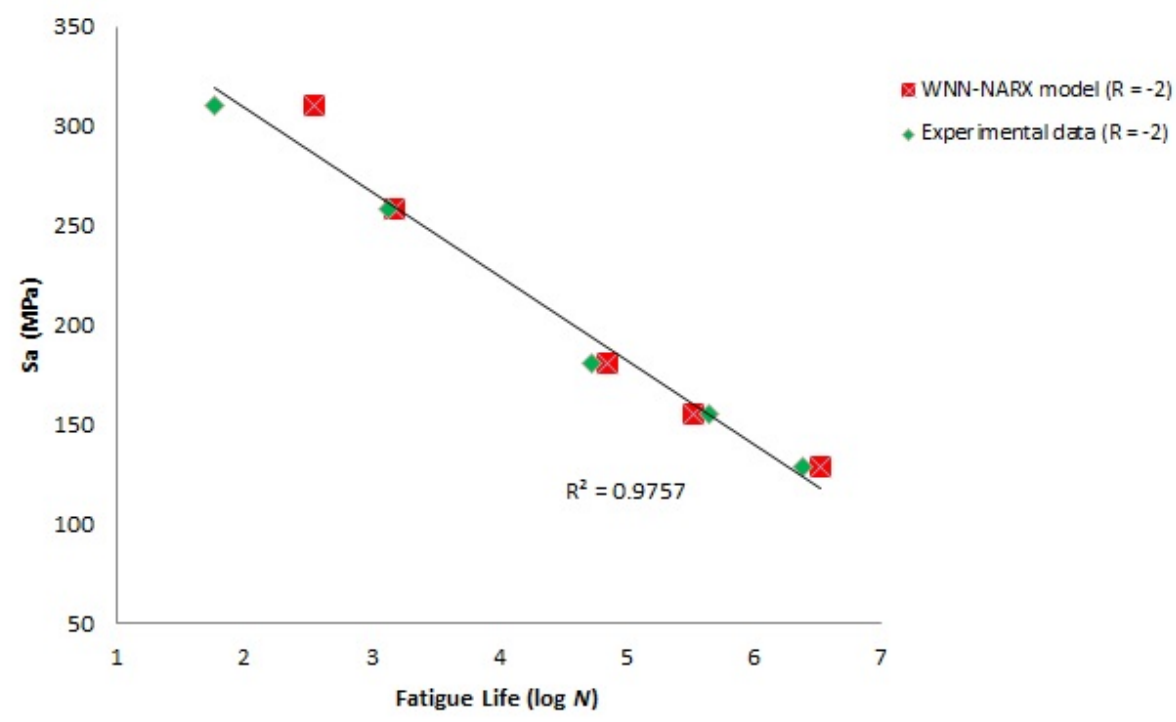

Fig. 7. Fatigue life prediction on the testing set of $R=-2$ for material 1 .

It is seen that the present hybrid WNN-NARX model produces fatigue life prediction results with good accuracies. The values of $\mathrm{R}^{2}$ range between 0.8549 and 0.9823 , where the lowest and highest $\mathrm{R}^{2}$ values are associated to the stress ratios $R=-0.5$ and $R=0.7$, respectively. For the fatigue life prediction results of the stress ratio $R=-0.5$, one can indeed observe larger discrepancies between the actual fatigue lives and the predicted ones. Nonetheless, in general the present hybrid WNN-NARX model shows its efficacy and capability for the fatigue life prediction of multivariable amplitude loadings. 


\subsection{Fatigue life prediction of material 2 (on-axis and off-axis)}

Figs. 8-11 depict fatigue life prediction results on the testing sets for material 2 of on-axis and off-axis, respectively.

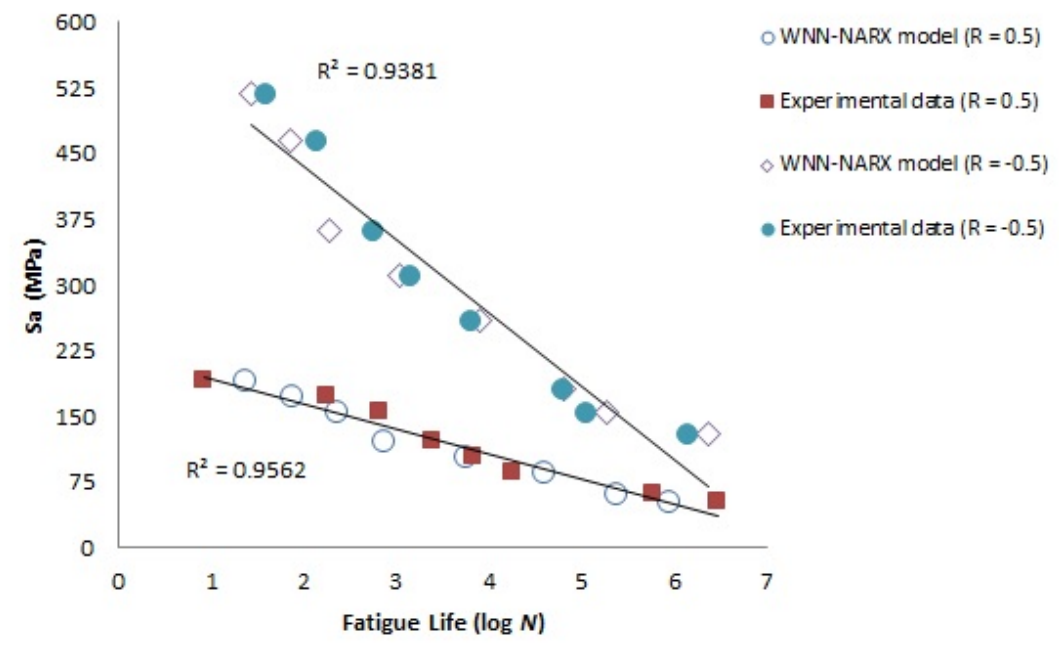

Fig. 8. Fatigue life prediction on the testing set of $R=0.5$ and -0.5 for material 2 (on-axis).

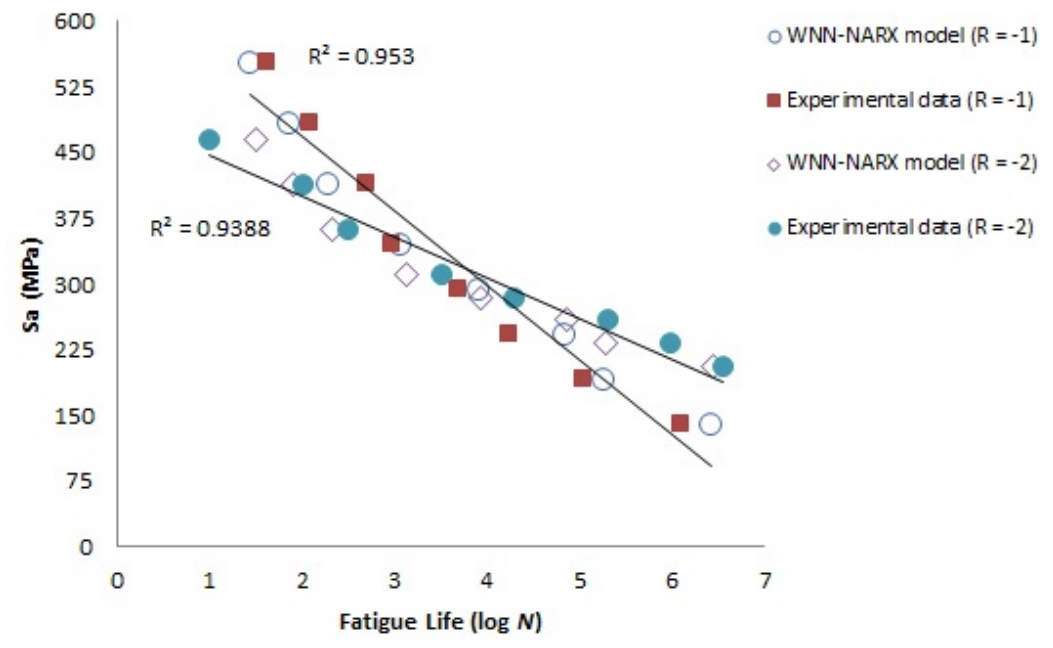

Fig. 9. Fatigue life prediction on the testing set of $R=-1$ and -2 for material 2 (on-axis). 


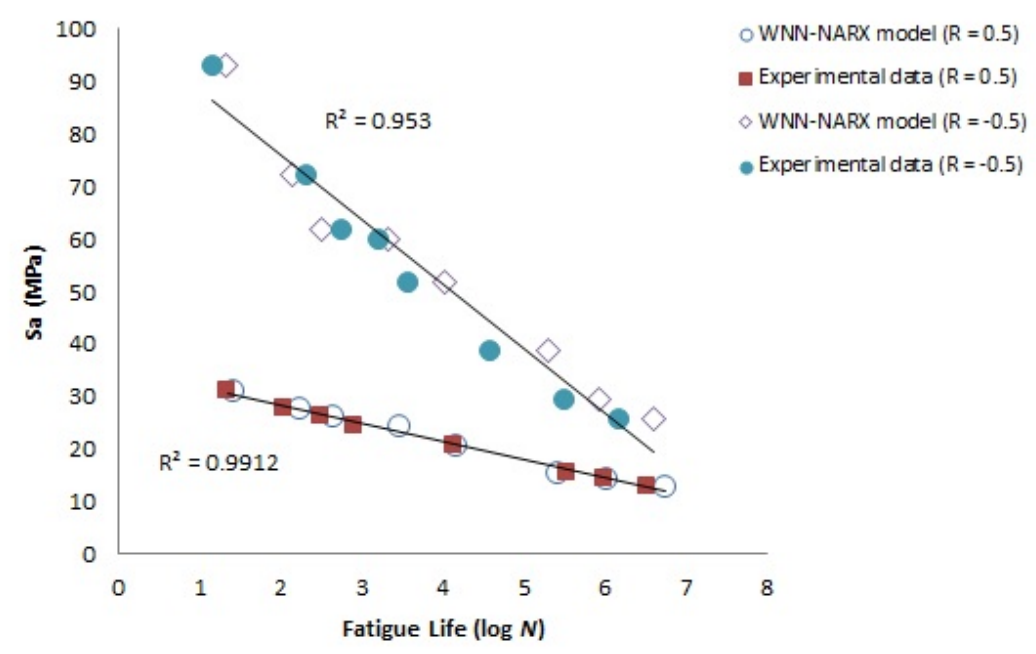

Fig. 10. Fatigue life prediction on the testing set of $R=0.5$ and -0.5 for material 2 (off-axis).

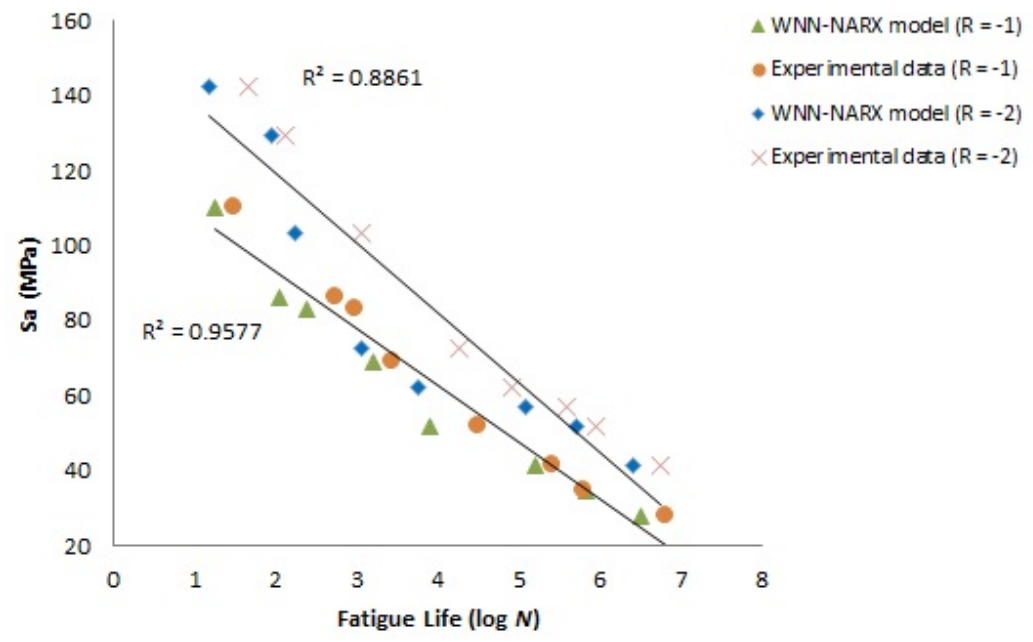

Fig. 11. Fatigue life prediction on the testing set of $R=-1$ and -2 for material 2 (off-axis).

It can be observed the present hybrid WNN-NARX model also produces fatigue life prediction results with good accuracies for the material. For material 2 on-axis, the values of $\mathrm{R}^{2}$ range from 0.9381 to 0.9562 . Moreover, the values range from 0.8861 to 0.9912 for material 2 off-axis. The lowest $\mathrm{R}^{2}$ value is associated to the stress ratio $R=-2$ for the material 2 off-axis. In general, the present hybrid WNN-NARX model is also suitable for the composite fatigue life prediction under variable amplitude loadings. 


\subsection{Fatigue life prediction of material 3 (on-axis and off-axis)}

Fatigue life prediction results on the testing sets for material 3 are depicted in Figs. 12 and 13.

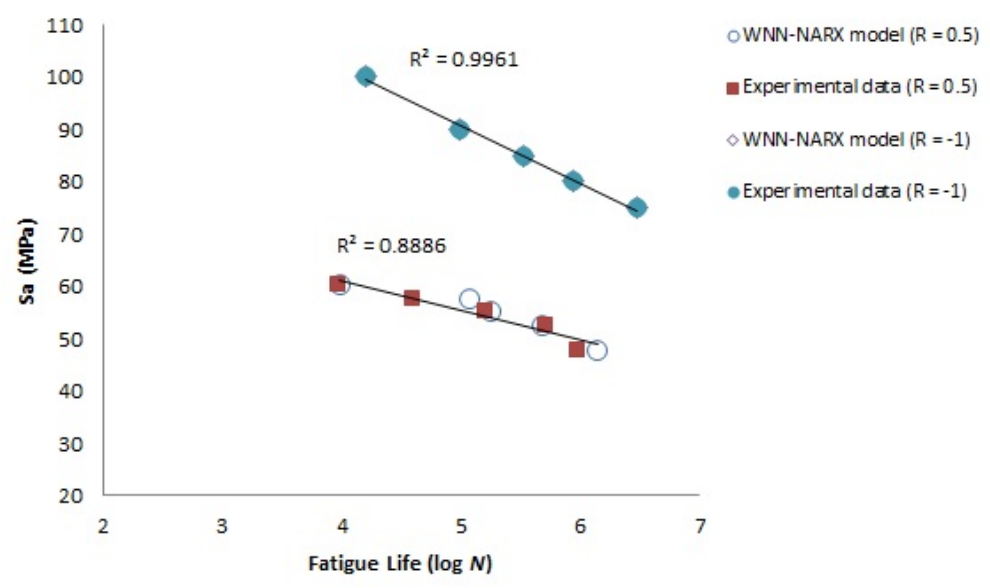

Fig. 12. Fatigue life prediction on the testing set of $R=0.5$ and -1 for material 3 (on-axis).

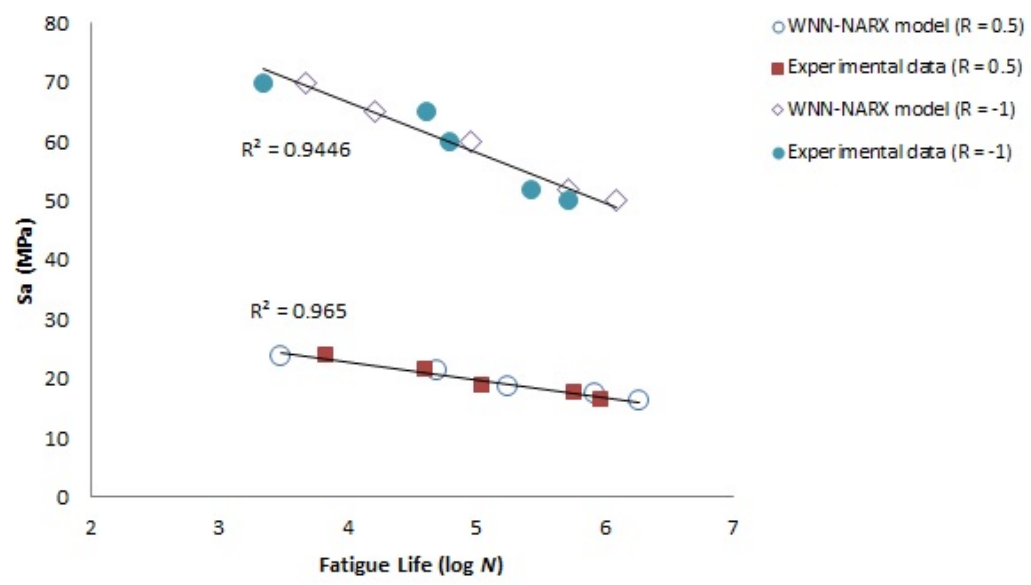

Fig. 13. Fatigue life prediction on the testing set of $R=0.5$ and -1 for material 3 (off-axis).

It can be seen that good accuracies are also obtained for the composite fatigue life prediction. High accuracy is even obtained for fatigue life prediction of the material 3 on-axis of $R=-1$. The values of $\mathrm{R}^{2}$ range from 0.8886 to 0.9961 and from 0.9446 to 0.965 for material 3 on-axis and material 3 off-axis, respectively. Larger discrepancies between the actual fatigue lives and the predicted ones are observed for the fatigue life prediction of material 3 on-axis at the stress ratio $R=0.5$. 


\subsection{Prediction interval of the composite fatigue life assessment}

Having the fatigue life assessment using NN, the prediction interval can be always obtained accordingly. In this section, the prediction intervals are only focused on and given for the fatigue life prediction results with lower $\mathrm{R}^{2}$ values. In Figs. 14-16, the prediction intervals are respectively shown for the prediction results on fatigue lives of material 1 at $R=-0.5\left(\mathrm{R}^{2}=0.8549\right)$, material 2 off-axis at $R=-2\left(\mathrm{R}^{2}=0.8861\right)$ and material 3 on-axis at $R=$ $0.5\left(R^{2}=0.8886\right)$.

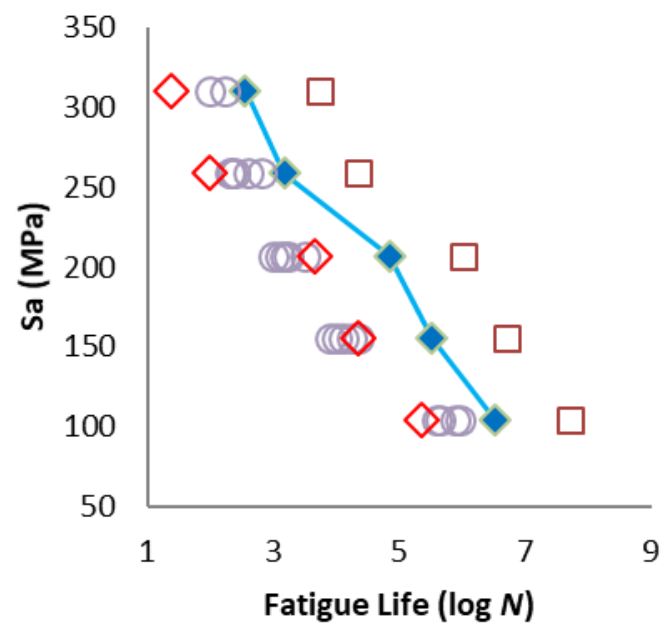

$-\rightarrow$ Mean lifetimes of WNN NARX model $(R=-0.5)$

- Scatter of experimental data $(R=-0.5)$

$\diamond$ Lower bounds of prediction

$\square$ Upper bounds of prediction

Fig. 14. Prediction interval of the WNN-NARX model for fatigue life assessment of material 1 of $R=-0.5$.

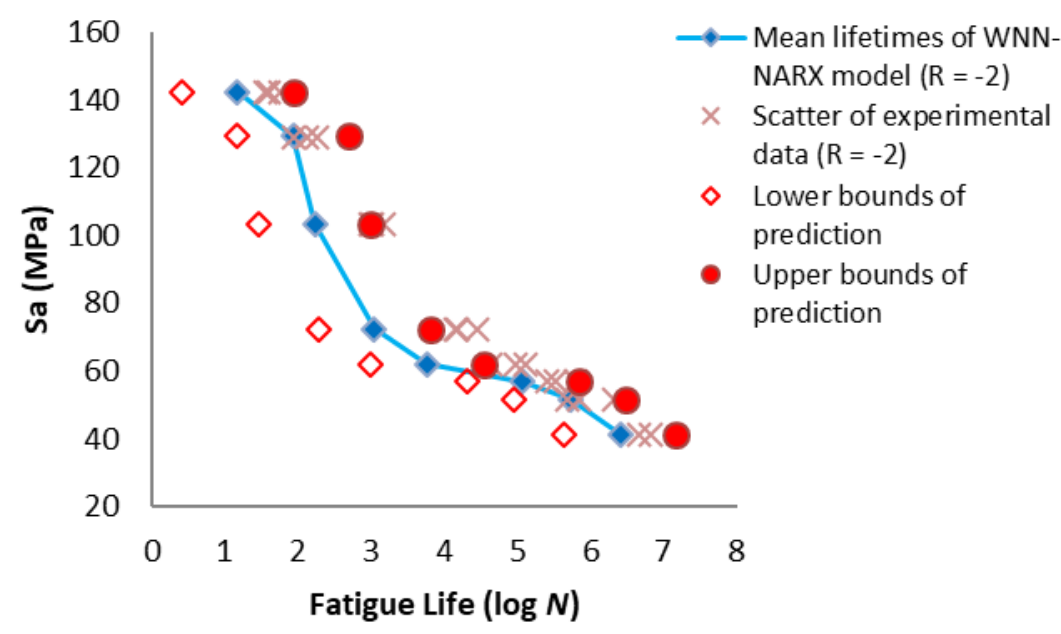

Fig. 15. Prediction interval of the WNN-NARX model for fatigue life assessment of material 2 off-axis of $R=-2$. 


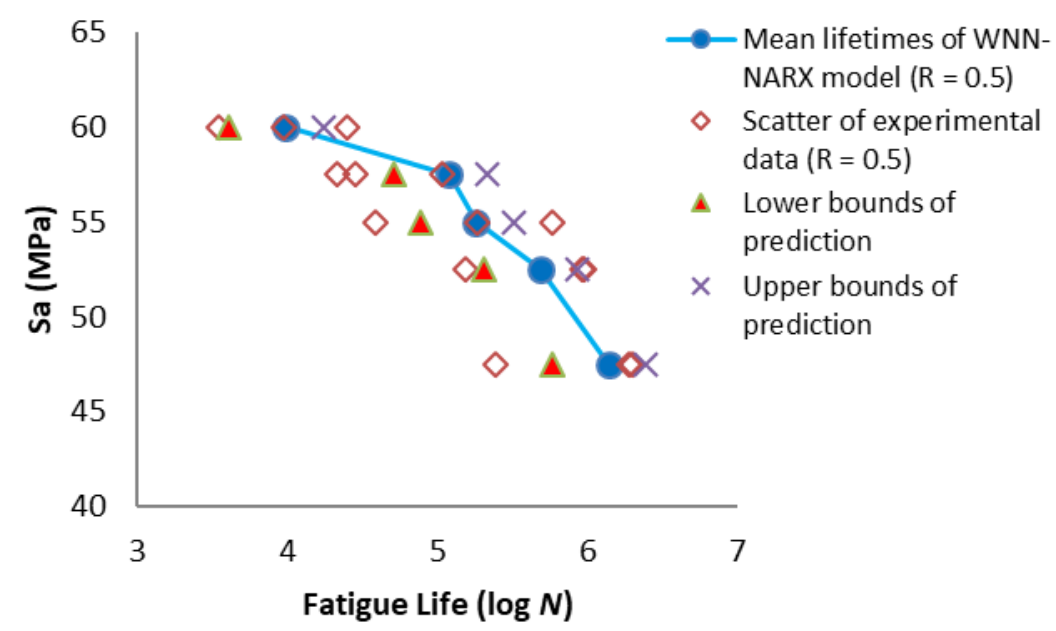

Fig. 16. Prediction interval of the WNN-NARX model for fatigue life assessment of material 3 on-axis of $R=0.5$.

The bounds of prediction shown in Figs. 14-16 are simply obtained from standard deviation of the prediction $\sigma$ given by:

with $n$ the number of data of interest.

$$
\sigma=\sqrt{\frac{\sum_{i=1}^{n}\left[y_{i}-\hat{y}_{i}\right]^{2}}{n-1}}
$$

It can be seen clearly that scatter of the fatigue lives of material 3 on-axis at $R=0.5$ is more pronounced or larger than that of the fatigue lives of material 1 at $R=-0.5$ and material 2 off-axis at $R=-2$, respectively. As a result, the fatigue lives of material 3 on-axis at $R=0.5$ are less contained within its bounds of prediction. On the other hand, the fatigue lives of material 1 at $R=-0.5$ and material 2 off-axis at $R=-2$ are more contained within their respective bounds of prediction. Scatter of the fatigue lives of material 1 at $R=-0.5$ is closer to its lower bound of prediction, while scatter of the fatigue lives of material 2 off-axis at $R=-2$ is closer to its upper bound of prediction. It appears that the scatter of fatigue lives would affect the accuracy of fatigue life prediction, considering that the fatigue life assessment is based upon mean lifetime values. Another factor seems to affect the fatigue life prediction accuracy is the position of stress ratio within the CLD. The aforementioned aspects are further discussed in the following section.

\subsection{Efficiency and accuracy of the composite fatigue life prediction}

The accuracy of prediction is measured by using the following error norm:

$$
\text { MSE }=\frac{\sum_{i=1}^{n}\left[y_{i}-\hat{y}_{i}\right]^{2}}{n}
$$

with $n$ is the number of data used in the prediction. To show the measure of prediction quality in overall, the MSE values for fatigue life prediction of composite materials by the present hybrid WNN-NARX model are shown in Table 4. 
Table 4. MSE of the fatigue life prediction by using the hybrid WNN-NARX predictive model

\begin{tabular}{cccccc}
\hline Materials & Training sets & Testing sets & $\begin{array}{c}\text { Range of } S_{\mathrm{m}} \\
\text { (MPa) }\end{array}$ & $\begin{array}{c}\text { Range of } S_{\mathrm{a}} \\
\text { (MPa) }\end{array}$ & $\begin{array}{c}\text { MSE of } \\
\text { prediction in } \\
\text { testing sets }\end{array}$ \\
\hline 1 & $R=0.1$ and 10 & $\begin{array}{c}R=0.9,0.8,0.7,0.5,-0.5, \\
-1 \text { and }-2\end{array}$ & -103.50 to 491.15 & 17.25 to 310.50 & 0.270 \\
2 (on-axis) & $R=0.1$ and 10 & $R=0.5,-0.5,-1$ and -2 & -155.25 to 568.50 & 51.75 to 552.00 & 0.125 \\
2 (off-axis) & $R=0.1$ and 10 & $R=0.5,-0.5,-1$ and -2 & -47.40 to 93.00 & 12.93 to 142.20 & 0.229 \\
3 (on-axis) & $R=0.1$ and 10 & $R=0.5$ and -1 & 0 to 180.00 & 47.5 to 100.00 & 0.194 \\
3 (off-axis) & $R=0.1$ and 10 & $R=0.5$ and -1 & 0 to 71.25 & 16.25 to 70.00 & 0.079 \\
\hline
\end{tabular}

From the presented results, it can be observed that the present hybrid WNN-NARX model produces reasonably accurate fatigue life prediction results by using fatigue data from two stress ratios. It is worth to note that the results improve the efficiency of fatigue life prediction of composite materials further. It is highlighted in the previous studies that accurate fatigue life prediction of composite materials can be achieved by employing $40-50 \%$ of the available data [9] or by employing training set of fatigue data from three S-N curves/stress ratios or more [13]. Improvement in the prediction is hence achieved in this study by using more limited stress ratio values, while the use of fatigue data from two stress ratios is justified profoundly, as already explained in the previous section. On the other hand, the use of two stress ratios also justifies the use of more limited fatigue data as training data i.e. $22.22 \%, 33.33 \%$ and $50 \%$ of the total fatigue data for the materials 1,2 and 3 respectively. In term of the percentage number of training data, the results are also consistent with those revealed in the previous study i.e. $40-50 \%$ of the available data [9], even though the previous study selected the training data directly from the available total data to get its percentage.

Therefore, the efficiency of fatigue life prediction is shown in the present study i.e. time and effort for collecting fatigue data of just two stress ratios can be more experimentally efficient and not too time consuming. In addition, having fatigue data from two $S-N$ curves in hands, one can proceed with the analysis and assessment for other fatigue lives. This in turn can give insights into other related aspects in design and speed up the design process of composite structures/components.

It is also shown that choosing two stress ratios to be used as the training data set is not too restrictive. Hence, there is a flexibility in choosing and collecting the fatigue data as training set, which further shows the robustness of the present fatigue life prediction. For more clarity of presentation, comparison of MSE values for the composite fatigue life prediction by using the present WNN-NARX model with different training data sets is further given in Table 5.

Table 5. Comparison of MSE values for the composite fatigue life prediction by using the present WNN-NARX model with different training data sets

\begin{tabular}{ccccccc}
\hline Materials & Training sets & $\begin{array}{c}\text { MSE of } \\
\text { prediction in } \\
\text { testing sets }\end{array}$ & Training sets & $\begin{array}{c}\text { MSE of } \\
\text { prediction in } \\
\text { testing sets }\end{array}$ & Training sets & $\begin{array}{c}\text { MSE of } \\
\text { prediction in } \\
\text { testing sets }\end{array}$ \\
\hline 1 & $R=0.1$ and 10 & 0.270 & $R=0.1$ and 0.5 & 0.264 & $R=0.1$ and -2 & 0.367 \\
2 (on-axis) & $R=0.1$ and 10 & 0.125 & $R=0.1$ and -2 & 0.211 & $R=0.1$ and -0.5 & 0.192 \\
2 (off-axis) & $R=0.1$ and 10 & 0.229 & $R=0.1$ and -2 & 0.241 & $R=0.1$ and -0.5 & 0.258 \\
3 (on-axis) & $R=0.1$ and 10 & 0.194 & $R=0.1$ and -1 & 0.294 & $R=0.1$ and 0.5 & 0.117 \\
3 (off-axis) & $R=0.1$ and 10 & 0.079 & $R=0.1$ and -1 & 0.115 & $R=0.1$ and 0.5 & 0.098 \\
\hline
\end{tabular}


Apart from the reasonably accurate fatigue life prediction results presented above, it is also important to notice the prediction results with larger discrepancies from the actual fatigue lives (lower $\mathrm{R}^{2}$ values). In general, it is observed that the larger discrepancies of prediction are associated to the stress ratio values which belong to tensile-compressive (T-C) and compressive-tensile (C-T) sectors in the CLD i.e. $R=-0.5$ and -2 . On the other hand, the composite fatigue life prediction for other stress ratios belong to tensile-tensile (T-T) and compressivecompressive (C-C) sectors are in general producing more accurate results.

It is interesting to also note that making stress ratios $R=-0.5,-1$ and -2 to be training data set seems not giving improvement on the prediction accuracy as well, as shown in Table 5. From the observation, it may be important to remark that further attention needs to be given to the corresponding fatigue life prediction results at the stress ratio values of tensile-compressive (T-C) and compressive-tensile (C-T) sectors in the CLD. In addition, attention also needs to be given to the fatigue life prediction results at the stress ratio $R$ with fatigue data exhibiting large scatter of fatigue lives, such as shown in Fig. 16 for material 3 on-axis of $R=0.5$. In short, larger discrepancies in fatigue life prediction of composite materials may be produced for those stress ratios. Although further exploration is still needed, to the present author's best knowledge this observation is still not highlighted and discussed in the previous research works on composite fatigue life prediction using NN. Moreover, it is hence necessary to refer to the prediction interval or bounds of prediction as discussed in the previous section so that comprehensive fatigue life assessment can be proceeded accordingly in such cases.

Further, the results show that fatigue life assessment of composite materials is still a challenging task. Further efforts are still required to get more comprehensive understanding on the relationship which might exist between failure mechanisms taking place in the composite materials at both micro and macro scales and the way of such mechanisms may be manifested in the corresponding fatigue data (such as the fatigue scatter), which might be possible to be captured further by some NN models. In addition, another interesting extension of study is that how to handle multivariable amplitude loadings, including multiaxial fatigue loadings, in a more integrated way in the fatigue life assessment using NN, other surrogate as well as combined forward (Hidayat et al., 2016; Hidayat et al., 2017; Hidayat et al., 2020) and inverse modelling techniques. These may be interesting as subjects of further study.

\section{Conclusions}

In this paper, hybrid WNN-NARX model has been developed and presented as data-driven based predictive model for efficient fatigue life assessment of composite materials under multivariable amplitude loadings. By observing fatigue data as dynamic data of stress ratio series, it is shown that the present hybrid WNN-NARX model produces reasonably accurate fatigue life prediction results by using fatigue data from two stress ratio values. In particular, the efficiency of composite fatigue life prediction by using $22.22 \%$ of total fatigue data can be achieved by using the hybrid WNN-NARX model. Several composite materials examined in this study show the efficacy and robustness of the proposed model. In addition, corresponding aspect of larger discrepancies between the actual fatigue lives and predicted ones in the composite fatigue life prediction which may be associated to certain stress ratio values have been also discussed. Such observation, still not explored in the previous research works, has given useful and integrated perspectives on fatigue life assessment of composite materials using NN. A more integrated way in the fatigue life assessment of composite materials using $\mathrm{NN}$ or other surrogate modelling techniques would be interesting as subject of further research study.

\section{Acknowledgments}

The supports from ITS Surabaya are gratefully acknowledged.

\section{References}

A. Bezazi, S. G. Pierce, K. Worden, and E. H. Harkati, "Fatigue life prediction of sandwich composite materials under flexural tests using a Bayesian trained artificial neural network," Int. J. Fatigue, 29(4), 738-747 (2007).

A. P. Vassilopoulos and T. P. Philippidis, "Complex stress state effect on fatigue life of GRP laminates. Part I, experimental. Int. J. Fatigue, 24(8), 813-823, (2002). 
A. P. Vassilopoulos, E. F. Georgopoulos, and T. Keller, "Comparison of genetic programming with conventional methods for fatigue life modeling of FRP composite materials,” Int. J. Fatigue, 30(9), 1634-1645 (2008).

A. Yaich, A. El Hami, L. Walha, and M. Haddar, "Local multiaxial fatigue damage estimation for structures under random vibrations,” Finite Elem. Anal. Des., 132, 1-7 (2017).

C. R. Kennedy, C. M. T. Brádaigh, and S. B. Leen, "A multiaxial fatigue damage model for fibre reinforced polymer composites,” Compos. Struct., 106, 201-210 (2013).

C.B. Kalayci, S. Karagoz and Ö. Karakas, "Soft computing methods for fatigue life estimation: A review of the current state and future trends”, Fatigue \& Fracture of Engineering Materials \& Structures, 143(12), 27632785 (2020).

D. Flore and K. Wegener, "Modelling the mean stress effect on fatigue life of fibre reinforced plastics," Int. J. Fatigue, 82, 689-699 (2016).

G.P. Sendeckyj, "Constant life diagrams-a historical review," Int. J. Fatigue, 23(4), 347-353 (2001).

H. Chen and D. G. Shang, "An on-line algorithm of fatigue damage evaluation under multiaxial random loading,” Int. J. Fatigue, 33(2), 250-254 (2011).

Harris, B. ed., 2003. Fatigue in Composites. Cambridge: Woodhead Publishing Ltd.

Hidayat, M.I.P. (2015). System Identification Technique and Neural Networks for Material Lifetime Assessment Application. In Q. Zhu \& A.T. Azar (Eds.), Complex System Modelling and Control Through Intelligent Soft Computations, Studies in Fuzziness and Soft Computing 319 (pp. 773-806). Switzerland: Springer International Publishing.

Hidayat, M.I.P., and Yusoff, P.S.M.M. (2009). Optimizing Neural Network Prediction of Composite Fatigue Life Under Variable Amplitude Loading Using Bayesian Regularization. In S. M. Sapuan \& I.M. Mujtaba (Eds.), Composite Materials Technology: Neural Network Applications (pp. 221-249). New York, USA: CRC Press.

I. Daubechies, Ten Lectures on Wavelets, Vol. 61, Society for Industrial and Applied Mathematics, Philadelphia, 1992, pp. 198-202.

J. C. F. Pujol and J. M. A. Pinto, “A neural network approach to fatigue life prediction,” Int. J. Fatigue, 33(3), 313-322 (2011).

J. Chen and Y. Liu, "Fatigue modeling using neural networks: a comprehensive review”, Authorea (2021). DOI: 10.22541/au.163254701.14733101/v1

J. D. Martin and T. W. Simpson, "Use of kriging models to approximate deterministic computer models," AIAA J., 43(4), 853-863 (2005).

J. F. Durodola, S. Ramachandra, S. Gerguri, and N. A. Fellows, “Artificial neural network for random fatigue loading analysis including the effect of mean stress,” Int. J. Fatigue, 111, 321-332 (2018).

J. F. Mandell and D. D. Samborsky, 2010. DOE/MSU composite material fatigue data-base: test, methods, material and analysis. SAND97-3002. Albuquerque, NM: Sandia National Laboratories.

J. Klemenc and M. Fajdiga, "Estimating S-N curves and their scatter using a differential ant-stigmergy algorithm. Int. J. Fatigue, 43(1), 90-97 (2012).

J. P. C. Kleijnen, “Kriging metamodeling in simulation: A review,” Eur. J. Oper. Res., 192(3), 707-716 (2009).

J. T. Hwang and J. R. R. A. Martins, "A fast-prediction surrogate model for large datasets," Aerosp. Sci. Technol., 75(2), 74-87 (2018).

J. Weng, W. Wen, and H. Zhang, "Multiaxial fatigue life prediction of composite materials," Chinese J. Aeronaut., 30(3), 1012-1020 (2017).

J. Y. Kang, B. I. Choi, H. J. Lee, J. S. Kim, and K. J. Kim, "Neural network application in fatigue damage analysis under multiaxial random loadings,” Int. J. Fatigue, 28(2), 132-140 (2006).

J. Yang, G. Kang, Y. Liu, K. Chen, and Q. Kan, "Life prediction for rate-dependent low-cycle fatigue of PA6 polymer considering ratchetting: Semi-empirical model and neural network based approach," Int. J. Fatigue, 136105619 (2020).

J. Zhu, W. Zhang, and X. Li, "Fatigue damage assessment of orthotropic steel deck using dynamic Bayesian networks,” Int. J. Fatigue, 118, 44-53 (2019).

J.F. Durodola, N. Li, S. Ramachandra, A.N. Thite, "A pattern recognition artificial neural network method for random fatigue loading life prediction," Int. J. Fatigue, 99, 55-67 (2017).

K. Narendra and K. Parthasarathy, "Identification and control of dynamic systems using neural networks," IEEE Trans. Neural Networks, 1(1), 4-27, (1990). 
L. K. Song, G. C. Bai, and C. W. Fei, "Probabilistic LCF life assessment for turbine discs with DC strategybased wavelet neural network regression,” Int. J. Fatigue, 119, 204-219 (2019).

M. Al-Assadi, H. A. E. Kadi, and I. M. Deiab, "Using artificial neural networks to predict the fatigue life of different composite materials including the stress ratio effect,” Appl. Compos. Mater., 18(4), 297-309 (2011).

M. J. Pais, F. A. C. Viana, and N. H. Kim, "Enabling high-order integration of fatigue crack growth with surrogate modeling,” Int. J. Fatigue, 43, 150-159 (2012).

M. Scheuerer, R. Schaback, and M. Schlather, "Interpolation of spatial data-A stochastic or a deterministic problem?” Eur. J. Appl. Math., 24(4), 601-629 (2013).

M.I.P. Hidayat, B.A. Wahjoedi and S. Parman, "B-spline collocation method for boundary value problems in complex domains", Int. J. Computing Science and Mathematics, 7(2), 110-125 (2016).

M.I.P. Hidayat, B.A. Wahjoedi, S. Parman and T.V.V.L.N. Rao, "Meshless Local B-Spline Collocation Method for Two-Dimensional Heat Conduction Problems With Nonhomogenous and TimeDependent Heat Sources”, Journal of Heat Transfer, 139, 071302-1 - 071302-11 (2017).

M.I.P. Hidayat, D.M. Felicia, F.I. Rafandi, A. Machmudah, "Effects of Sample Shapes and Thickness on Distribution of Temperature inside the Mineral Ilmenite Due to Microwave Heating", Minerals, 10(4):382, 1-19 (2020).

N. Stojković, R. Folić, and H. Pasternak, "Mathematical model for the prediction of strength degradation of composites subjected to constant amplitude fatigue,” Int. J. Fatigue, 103, 478-487 (2017).

Neural Network Toolbox ${ }^{\mathrm{TM}}$ User's Guide (C) COPYRIGHT 1992-2017, The MathWorks, Inc., USA.

P. Prebeg, V. Zanic, and B. Vazic, “Application of a surrogate modeling to the ship structural design," Ocean Eng., 84, 259-272 (2014).

Q. Zhang and A. Benveniste, “Wavelet Networks,” IEEE Trans. Neural Networks, 3(6), 889-898 (1992).

R. K. Tripathy and I. Bilionis, "Deep UQ: Learning deep neural network surrogate models for high dimensional uncertainty quantification,” J. Comput. Phys., 375, 565-588 (2018).

R.B. Kalombo, M.S. Pestana, R.C.S. Freire Júnior, J.L.A. Ferreira, C.R.M. Silva, L.A.C.M. Veloso, E.C.B. Câmara, J.A. Araújo, "Fatigue life estimation of an all aluminium alloy 1055 MCM conductor for different mean stresses using an artificial neural network," Int. J. Fatigue, 140105814 (2020).

R.C.S. Freire Junior, A.D.D. Neto and E.M.F. de Aquino, "Building of constant life diagrams of fatigue using artificial neural networks," Int. J. Fatigue, 27(7), 746-751 (2005).

Reifsnider, K.L. ed., 1991. Fatigue of Composite Materials. Amsterdam: Elsevier.

S. Chen, S. A. Billings and P. M. Grant, "Non-linear system identification using neural networks." Int. J. Control, 51(6), 1191-1214 (1990).

S. Shiri, M. Yazdani, and M. Pourgol-Mohammad, "A fatigue damage accumulation model based on stiffness degradation of composite materials,” Mater. Des., 88, 1290-1295 (2015).

S.G. Mallat, "A theory for multiresolution signal decomposition: the wavelet representation," IEEE Trans. Pattern Anal. Mach. Intell," 11(7), 674-693 (1989).

Schijve, J. ed., 2009. Fatigue of Structures and Materials. Netherlands: Springer.

T. Bučar, M. Nagode, and M. Fajdiga, "A neural network approach to describing the scatter of S-N curves," Int. J. Fatigue, 28(4), 311-323 (2006).

V. Shanmugam, O. Das, K. Babu, U. Marimuthu, A. Veerasimman, D. J. Johnson, R. E. Neisiany, M. S. Hedenqvist, S. Ramakrishna and F. Berto, "Fatigue behaviour of FDM-3D printed polymers, polymeric composites and architected cellular materials," Int. J. Fatigue, 143106007 (2021).

W. Lian and W. Yao, "Fatigue life prediction of composite laminates by FEA simulation method," Int. J. Fatigue, 32(1), 123-133 (2010).

Z.-H. Han, K.-S. Zhang, W.-P. Song and Z.-D. Qiao, “Optimization of active flow control over an airfoil using a surrogate-management framework,” J. Aircr., 47(2), 603-612 (2010). 